\title{
DHPG activation of group 1 mGluRs in BLA enhances fear conditioning
}

\author{
Jerry W. Rudy ${ }^{1}$ and Patricia Matus-Amat \\ Department of Psychology, Center for Neuroscience, University of Colorado, Boulder, Colorado 80309, USA
}

\begin{abstract}
Group 1 metabotropic glutamate receptors are known to play an important role in both synaptic plasticity and memory. We show that activating these receptors prior to fear conditioning by infusing the group $1 \mathrm{mGluR}$ agonist, (R.S.)-3,5dihydroxyphenylglycine (DHPG), into the basolateral region of the amygdala (BLA) of adult Sprague-Dawley rats enhances freezing normally supported by a weak footshock. This effect of DHPG was blocked when it was co-infused with either the general group 1 mGluRI antagonist, (R,S)-1-aminoindan-1,5 dicarboxylic acid (AIDA), or with the selective mGluR5 antagonist, 2-methyl-6-(phenylethynyl)-pyridine (MPEP). These results support previous findings by Rodrigues and colleagues that mGluR5s in the lateral region of the amygdala make an import contribution to fear conditioning. More importantly, they support the general ideas embedded in the concept of metaplasticity, as per Abraham, and the synaptic-tagging hypothesis per Frey and Morris-that the processes that specify the content of experience can be experimentally separated from those needed to acquire the memory.
\end{abstract}

[Supplemental material is available online at www.learnmem.org.]

The last decade has seen an increased appreciation of the view that the plasticity state of neurons-their ability to be modified by experience-is not fixed. Instead, the effect of experience depends on the physiological or biochemical state of the neurons or synapses that receive and store information contained in the experience, and this state is variable. This perspective is captured by the concept called "metaplasticity" (Abraham and Bear 1996; Abraham and Tate 1997; Abraham 2008), which recognizes that prior events can change the general plasticity or modifiability of neurons and synapses that will potentially store information contained in a subsequent target experience. This idea is also embedded in the synaptic-tagging hypothesis (Frey and Morris 1997, 1998) that will be described in some detail in the Discussion section. This view is important because it recognizes that the processes that specify the content of experience can be experimentally separated from those that are needed to store the memory for the experience.

As reviewed by Abraham (2008), the range of prior events that can potentially determine a neuron's state of plasticity is quite large and can be mediated by their effects on many components of the machinery that supports changes in synaptic strength. They include modification in NMDA-receptor function, AMPA-receptor trafficking, neuronal excitability, and epigenetic modifications.

One way that the potential for plasticity can be altered is by activation of group 1 metabotropic glutamate receptors (mGluR1s and mGlur5s) (Abraham 2008). A number of reports based on the in vitro long-term potentiation (LTP) methodology suggest that activating this class of receptors prior to the delivery of a relatively weak inducing stimulus can transform a normally short-lasting form of LTP into a more persistent form (Cohen and Abraham 1996; Cohen et al. 1998; Raymond et al. 2000). For example, an infusion of (R.S.)-3,5-dihydroxyphenylglycine (DHPG), a group $1 \mathrm{mGluR}$ agonist, into the bathing medium $30 \mathrm{~min}$ prior to the delivery of a weak inducing stimulus can significantly enhance the persistence of the resulting LTP, while having no effect on the baseline response (Cohen et al. 1998; Raymond et al. 2000). This effect of DHPG, however, is blocked (Raymond et al. 2000) when its

'Corresponding author.

E-mail JRudy@Clipr.Colorado.edu; fax (303) 492-2967.

Article is online at http://www.learnmem.org/cgi/doi/10.1101//m.1444909. administration is accompanied by an infusion of the group $1 \mathrm{mGluR}$ antagonist, (R,S)-1-aminoindan-1,5 dicarboxylic acid (AIDA).

Group 1 mGluRs also have been implicated in fear conditioning. For example, Rodrigues et al. (2002) reported that one subtype, mGluR5, is localized in dendrites and spines in neurons in the lateral nucleus of the amygdala, and fear conditioning can be significantly impaired if the mGluR5 antagonist, 2-methyl-6(phenylethynyl)-pyridine (MPEP), is infused into the lateral nucleus prior to conditioning.

These findings, from the studies of synaptic plasticity and fear conditioning, provide the empirical basis for the hypothesis that motivates the experiments reported here-that the plasticity potential of neurons in the amygdala that support fear conditioning can be increased by activating group 1 mGluRs prior to the conditioning experience.

To evaluate this idea, we followed a strategy similar to that used to study the role of mGluRs in LTP (Cohen and Abraham 1996; Cohen et al. 1998; Raymond et al. 2000). In these in vitro LTP experiments, a relatively weak inducing stimulus was used to generate a short-lasting LTP. Infusing the group 1 agonist DHPG prior to the delivery of the inducing stimulus transformed this short-lasting LTP into a more persistent form. Their results suggest that the activation of group 1 mGluR1 receptors independently initiates processes that make an important contribution to longlasting LTP. To apply this strategy to fear conditioning, we used a very low level of shock-one that by itself produced an almost undetectable level of conditioned fear (as measured by freezing, the innate defensive response of rodents). We then determined if the activation of group 1 mGluRs prior to the conditioning experience would transform this outcome and produce a stronger level of freezing. DPHG was infused into the basolateral region of the amygdala (BLA) to activate the mGluRs.

\section{Results}

\section{Histology}

In all experiments the intent was to inject the drug into the BLA. Rats were eliminated from the experiment if either of the cannula track marks was anterior to $-2.30 \mathrm{~mm}$ or posterior to $-3.6 \mathrm{~mm}$; 
lateral to $\pm 5.5 \mathrm{~mm}$ or medial to $\pm 4.5 \mathrm{~mm}$; or dorsal to $-7.0 \mathrm{~mm}$ or ventral to $-9.0 \mathrm{~mm}$. Cannula locations for all experiments are presented in Supplemental Figures 1-4.

\section{Experiment 1. DHPG enhances long-term fear conditioning produced by weak shock}

DHPG $(n=13)$ or vehicle $(n=14)$ was infused into the amygdala $\sim 30$ min prior to fear conditioning. Two minutes after rats were placed into the conditioning chamber, an auditory cue was presented for $15 \mathrm{sec}$ and terminated with the delivery of a 2-sec $0.35-\mathrm{mA}$ shock. Rats were then returned to their home cage. Approximately $24 \mathrm{~h}$ later the rats were tested for fear of the context and then returned to their home cage. Approximately $2 \mathrm{~h}$ later rats were placed into a different context where they were tested for fear of the tone. The dependent variable was the rat's innate defensive response, freezing.

As shown in Figure 1, DHPG enhanced freezing to both the context, $F_{(1,25)}=4.6, P<0.04$, and to the auditory-cue fear test, $F_{(1,25)}=9.7, P<0.005$. These results are consistent with the hypothesis that activating group $1 \mathrm{mGluR}$ receptors prior to the conditioning experience can enhance plasticity potential.

\section{Experiment 2. DHPG alone does not increase freezing}

The results of Experiment 1 support the hypothesis that activating the group 1 mGluRs enhances the plasticity of neurons in the amygdala that support the fear memory. This conclusion, however, assumes that the increased level of freezing displayed by rats injected with DHPG depended on the combination of pretreatment with DHPG and the conditioning treatment. It is possible, however, that the enhanced freezing was simply a consequence of pretreatment with DHPG prior to exposure to just the context and the auditory cue. To rule this out, rats were pretreated with DHPG $(n=8)$ or its vehicle $(n=8)$ and then exposed to the conditioning context and auditory cue. The next day rats were tested for their response to the context and the auditory cue. As expected, neither the rats injected with the vehicle or the drug displayed any freezing. The mean level of freezing was $<1 \%$ for both sets of animals, $F_{(1,14)}<1.0$. Thus, the application of DHGP alone has no effect on subsequent test behavior.

\section{Experiment 3. DHPA delivered 6 but not $24 \mathrm{~h}$ prior to training enhances fear conditioning}

DHPG facilitates fear conditioning when it is delivered $30 \mathrm{~min}$ prior to training. To place some boundary conditions on this result, in this experiment DHPG ( $n=6$ and $n=8)$ or vehicle $(n=13)$ was injected into the BLA either 6 or $24 \mathrm{~h}$ prior to just contextual fear conditioning. Figure 2 shows that DHPG enhanced freezing when it was injected $6 \mathrm{~h}$ prior to training, $F_{(2,24)}=5.2, P<0.01$. Post-hoc

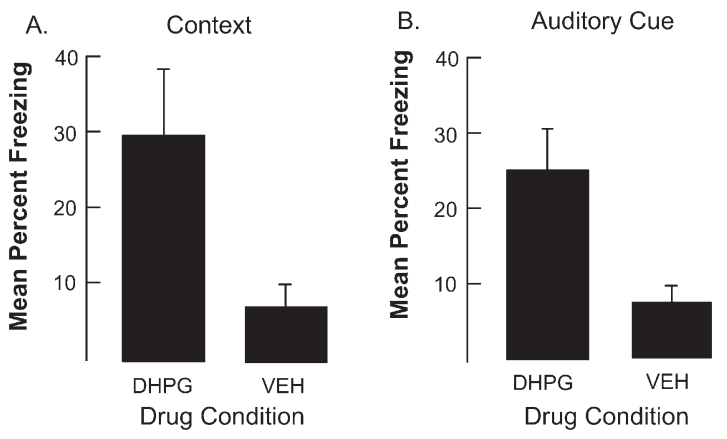

Figure 1. The results of Experiment 1. Panel $A$ displays freezing during the context test. Panel $B$ displays freezing during the auditory cue test. Note that injecting DHPG into the BLA enhanced freezing during both contextual and auditory-cue tests. Bars, SE.

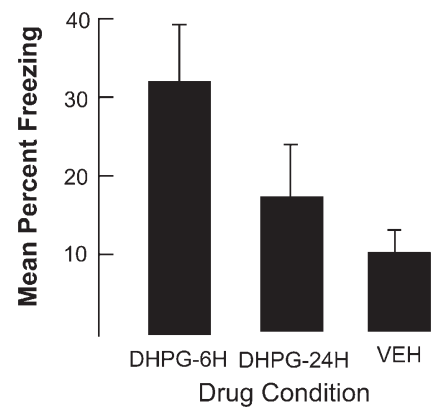

Figure 2. The results of Experiment 2. Note that injecting DHPG into the BLA $6 \mathrm{~h}$ but not $24 \mathrm{~h}$ prior to contextual fear conditioning enhanced freezing during the contextual fear test. Bars, SE.

comparisons revealed that only the rats injected with DHPG $6 \mathrm{~h}$ prior to conditioning differed from rats injected with the vehicle $(P<0.01)$. No other differences achieved the conventional $0.05-$ confidence level of significance, although the difference between the rats in the 6- and 24-h DHPG conditions approached this level.

\section{Experiment 4. Enhanced freezing associated with DHPG is blocked by AIDA}

We assume that enhanced freezing associated with DHPG is mediated by its effect on group 1 mGluRs. If this is true, then it should be possible to block the effect by co-treatment with the group 1 mGluR antagonist, AIDA. As shown in Figure 3, compared to the vehicle $(n=9)$, DHPG alone $(n=12)$ enhanced freezing to both the context and to the auditory cue, but this effect was blocked by AIDA $(n=12)$. The analyses of variance on the context and auditory cue data were both significant, $F \mathrm{~s}_{(2,30)}=3.8$ and 4.6 , $P s<0.03$. Post-hoc comparisons indicate that rats treated with DHPG were significantly different from rats in the other two conditions $(P \mathrm{~s}<0.02)$. These results are consistent with the hypothesis that the facilitating effect of DHPG on fear conditioning is mediating via its agonizing effect on group 1 mGluRs.

\section{Experiment 5. Enhanced freezing associated with DHPG is blocked by MPEP}

As noted, Rodrigues et al. (2002) provided evidence that the group 1 mGluR receptor subtype, mGluR5, contributes to the memory for a fear conditioning experience. In contrast, Kim et al. (2007) found no evidence that the group 1 subtype, mGluR1, contributes to fear conditioning, although they did find that blocking these receptors impaired the extinction of the fear response. These findings suggest that the effects of DHPG were mediated by the activation of the mGluR5 subtype. To evaluate this hypothesis, we repeated the previous experiment but combined DPHG with 2-methyl-6-(phenylethyl)-pyridine (MPEP), a specific mGluR5 antagonist (Rodrigues et al. 2002), to examine the role of mGluR5 in contextual fear conditioning.

As shown in Figure 4, the enhanced freezing produced when DHPG was injected into the BLA prior to conditioning was eliminated when it was co-administered with MPEP. An ANOVA revealed significant differences among the groups, $F_{(2,11)}=12.8, P<$ 0.001. Post-hoc comparisons indicated that rats in the DHPG condition differed from the other two conditions $(P s<0.002)$. No other differences were significant. These results are consistent with the hypothesis that group 1 mGluR5 receptors mediate the effects of DHPG that are associated with enhanced fear conditioning.

\section{Discussion}

Our experiments revealed that injecting DHGP into the BLA prior to fear conditioning significantly enhanced subsequent freezing 
A.

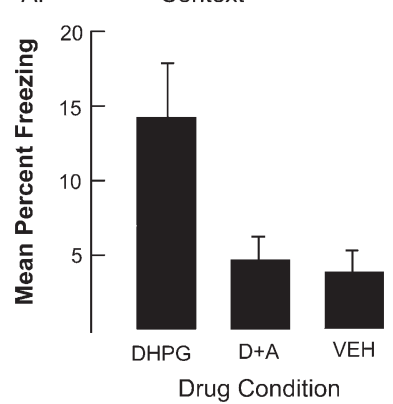

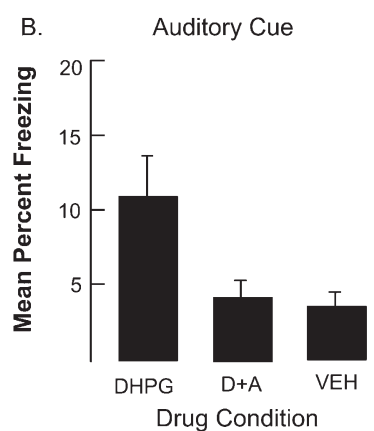

Figure 3. The results of Experiment 3. Panel $A$ displays freezing during the context test. Panel $B$ displays freezing during the auditory cue test. Note that AIDA co-injected with DHPG eliminated enhanced freezing associated with DHPG alone. Bars, SE. Key: D+A = DHPG + AIDA.

to both context and the auditory cue paired with a weak shock. This effect of DHPG depended on the rats being shocked because the drug did not enhance freezing displayed by rats that experienced the context and tone but were not shocked. Moreover, there was a temporal dependency to the effect of DHPG on contextual fear conditioning - it enhanced fear conditioning when injected 1 and $6 \mathrm{~h}$ before training but not when injected $24 \mathrm{~h}$ before training. Both the general group $1 \mathrm{mGluR}$ antagonist AIDA and the selective mGluR5 antagonist MPEP prevented the enhancing effect of DHPG. Thus, enhanced freezing produced by DHGP appears to be the result of its activating group $1 \mathrm{mGluRs}$ and more specifically mGluR5s.

A number of studies have implicated group 1 mGluRs, especially mGluR5, in both synaptic plasticity (Huber et al. 1998; Balschun et al. 1999; Raymond et al. 2000) and memory (Lu et al. 1997; Bortolotto et al. 1999; Reidel et al. 2000; Rodrigues et al. 2002). Much of this evidence is derived from studies of synaptic plasticity in the hippocampus and studies with memory tasks that depend on the hippocampus. However, Rodrigues et al. (2002) have made a strong case that mGluR5s in the lateral amygdala (LA) also play an important role in synaptic plasticity and the formation of fear memories. They conditioned rats with a relatively strong shock that by itself produced significant fear conditioning. However, an injection of the mGluR5 antagonist MPEP into the LA significantly reduced the level of freezing, suggesting that mGluR5s were activated by the strong shock and contributed to the strength of the fear response. Their work also revealed that mGluR5s are localized to dendritic shafts and spines in the LA, and that LTP at synaptic inputs to the LA is impaired by MPEP.

Our results are consistent with those reported by Rodrigues et al. (2002) and they support the hypotheses that mGluR5s in the LA make an important contribution to fear conditioning. To make this clear, it is easy to produce an enduring fear memory when a relatively intense single shock is used, as was the case in the Rodrigues et al. (2002) experiment. Theoretically, a strong shock activates both the processes needed to specify the content of the memory and the processes, such as those controlled by group 1 mGluR5s that are needed to produce a strong fear memory. In contrast, in our experiments the weak shock is sufficient to specify content but not sufficient to generate an enduring fear memory because theoretically it does not activate group 1 mGlur5s. However, this weakness can be ameliorated by independently activating these receptors with DPHG.

Our experiments were modeled after those used to study the role of mGluRs in LTP (Cohen and Abraham 1996; Cohen et al. 1998; Raymond et al. 2000). These experiments revealed that a short-lasting LTP produced by a relatively weak inducing stimulus could be converted into a longer-lasting LTP through an infusion of DHPG. Importantly, in our experiments DPHG also enhanced the expression of a fear memory, just as it enhanced the LTP.

Raymond et al. (2000) proposed that DHPG activation of this class of mGluRs results in the local synthesis of new proteins (see Steward and Schuman 2001; Bradshaw et al. 2003; Sutton and Schuman 2006) that can then be used to produce a longlasting LTP. This conclusion was based on their findings, using the hippocampal-slice preparation, that the ability of DHPG to produce a long-lasting LTP was blocked by the protein synthesis inhibitor emetine, whereas the transcription inhibitor actinomycin D did not prevent this effect. Abraham (2008) also has suggested local protein synthesis as an outcome initiated by activating mGluRs that could enhance the duration of LTP. It is possible that local synthesis initiated by DHPG also contributes to the behavioral results we report.

It is interesting to speculate on a potential relationship between our results and the synaptic-tagging hypothesis proposed by Frey and Morris (1997, 1998; see also Frey and Frey 2008). They proposed that synaptic stimulation provided by an LTP-inducing stimulus engages two sets of separable processes. One set tags specific synapses and thus supports the content of a particular experience, and another set of intracellular processes makes available so-called "plasticity products" (PPs)—new transcripts and proteins-needed to secure a long-lasting LTP. Synaptic tags can be produced by relatively weak synaptic inputs, but the generation of PPs requires strong stimulus input. Both tags and plasticity products decay with time. However, when the tag overlaps with the availability of PPs it can capture these products, and an enduring LTP can be established at those synapses. Otherwise, the once potentiated synapses will return to their baseline state.

Frey and Morris (1997) provided the first evidence that the tagging and PP generation properties of synaptic stimulation are independent. They showed that weak stimulation to one set of synapses could produce long-lasting LTP if strong stimulation was applied to other synapses on the same neuron, either shortly before or after weak stimulation. There are many examples supporting the idea that the PP generation processes needed for long-lasting LTP can be engaged independent of the processes that specify the content of the experience (e.g., Dudek and Fields 2002; Foneseca et al. 2004; Alarcon et al. 2006; Sajikumar et al. 2007; for reviews, see Reymann and Frey 2007; Frey and Frey 2008).

However, the previously described findings of Cohen and Abraham (1996), Cohen et al. (1998), and Raymond et al. (2000) are the most relevant. Recall that they reported that the application of DPHG to bathing medium containing a hippocampal slice preparation can significantly enhance the duration of LTP normally produced by a weak inducing stimulus. This finding suggests that activating group 1 mGluRs initiates an intracellular

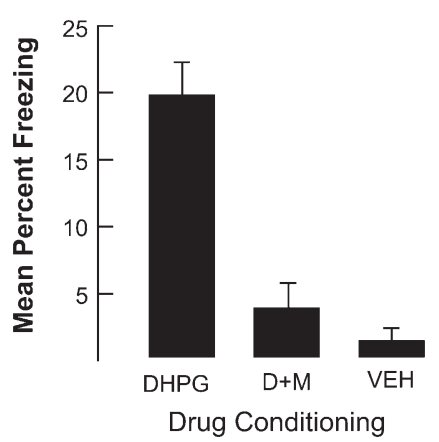

Figure 4. The results of Experiment 4. Note that MPEP co-injected with DHPG eliminated enhanced freezing associated with DHPG alone. Bars, SE. Key: D+M = DHPG + MPEP. 
cascade that produces PPs (needed to support long-lasting LTP) that are captured by synapses stimulated by the weak stimulus input.

Our behavioral experiments also suggest a segregation of the tagging-like processes and processes that generate PPs. In this case, the fear conditioning experience involving a weak shock is sufficient to specify content but not sufficient to generate the PPs needed to produce an enduring fear memory because it does not activate group 1 mGlur5s. However, independently activating these receptors with DPHG might then produce the plasticity products that support a lasting fear memory.

Although the synaptic-tagging hypothesis provides a plausible framework for interpreting our results, it should be appreciated that its application to the present data is speculative and that there are other ways in which DHPG might produce its effect. For example, it might increase the overall excitability of the neurons in the LA region so that the relative weak shock itself is responded to as if it were more intense.

In summary, injecting DPHG into the BLA significantly enhanced fear conditioning produced by weak shock. This effect was blocked by the co-injection of either AIDA, a nonselective group 1 mGluR antagonist, or MPEP, a selective mGluR5 antagonist. These results support the hypothesis that group 1 mGluR receptors in the BLA make an important contribution to fear conditioning (Rodrigues et al. 2002). More importantly they support the more general ideas embedded in the concept of metaplasticity (Abraham 2008) and the synaptic-tagging hypothesis (Frey and Morris 1998) - that the processes that specify the content of experience can be experimentally separated from those needed to acquire the memory.

\section{Materials and Methods}

\section{Subjects}

Adult male Sprague-Dawley rats weighing 250-300 g at the time of surgery were housed to a cage at $25^{\circ} \mathrm{C}$ on a 12 -h light/12-h dark cycle (lights on at 7:00 a.m.). Rats were allowed free access to food and water. Rats were given $1 \mathrm{wk}$ to acclimate to colony conditions before experimentation began. All experiments were conducted in accordance with protocols approved by the University of Colorado Animal Care and Use Committee.

\section{Surgery}

Under halothane anesthesia, rats were placed into a stereotaxic apparatus (David Kopf Instruments), and stainless steel guide cannulae (Plastics One) were implanted bilaterally at the BLA. On the basis of the Paxinos and Watson (1998) rat brain atlas, the following coordinates were used for bilateral BLA implantation: AP: $-2.8 \mathrm{~mm}$; ML: $4.8 \mathrm{~mm}$; DV: $-7.5 \mathrm{~mm}$ relative to bregma. Cannulae were fixed to the skull with dental acrylic and three small screws. To maintain patency, after surgery we placed an obturator into, and extended $1 \mathrm{~mm}$ beyond the tip of, the guide cannula (total length, $8.5 \mathrm{~mm}$ for BLA). Rats were allowed to recover for 7-10 d before being trained on the behavioral task.

\section{Apparatus}

Auditory/contextual cued fear conditioning and testing were carried out in two identical Igloo ice chests $(54$ long $\times 30$ wide $\times 27$ high, $\mathrm{cm}$ ) with white interiors. The conditioning chambers $(26$ long $\times 21$ wide $\times 24$ high, $\mathrm{cm}$ ) placed inside each chest were made of clear plastic and had window screen tops. A speaker and an activated 120-V (AC), 6-W light bulb were mounted on the ceiling of each chest. The ice chest door was open the entire time, and the room was illuminated by two 60 -W light bulbs. The 2 -sec 0.35 or $0.5 \mathrm{~mA}$ shock was delivered through a removable floor of stainless steel rods (Model E63-23 MMOD001; Coulbourn Instruments), each of which was $0.5 \mathrm{~cm}$ in diameter and spaced $1.75 \mathrm{~cm}$ center to center. Each rod was wired to a shock generator and scrambler (Model H13-16, Coulbourn Instruments). The chamber was cleaned with water before each rat was conditioned or tested.

\section{Behavioral procedures}

Rats were taken two at a time from their home cage and transported to the conditioning room in a black ice bucket. The lid was on so that the rats could not see where they were being taken. The rats were placed into the conditioning chamber and 2 min later received one presentation of a $15-\mathrm{sec}, 76 \mathrm{~dB}, 2000-\mathrm{Hz}$ tone. The tone terminated in a 2 -sec, $0.35 \mathrm{~mA}$ shock. Following the shock, rats were immediately removed from the chambers, and returned to the home cage. The next day, all rats received a 6-min test to determine their level of freezing (the rat's natural response to anticipated danger) (Blanchard and Blanchard 1969) to just the context. Approximately $3 \mathrm{~h}$ later, these rats received an auditorycue fear test in a novel environment. Three minutes after the rat was placed into the novel context the tone was presented for 3 min. To create a novel test context, the shock grid was removed from the chamber, and the original test was replaced with a novel test chamber $(26 \mathrm{~cm}$ long $\times 21 \mathrm{~cm}$ wide $\times 10 \mathrm{~cm}$ high) between two diagonally opposite corners, forming a triangular chamber. The chamber sat on a Plexiglas floor, and a 7-W, 120 VAC clear red light bulb illuminated the Igloo chest. The intensity of the light was reduced just to the point at which the observers could still score the behavior. There was no other lighting in the test room. Freezing was scored during the 3-min tone presentation using the sampling procedure described above.

In all experiments, freezing was the measure of conditioned fear. Using a sampling procedure, each rat was judged as either freezing or active every $10 \mathrm{sec}$ at that instant. Freezing was defined as the absence of all movement except for respiration. The test period lasted $5 \mathrm{~min}$. All scoring was conducted by an experimental observer who had no knowledge of the rat's treatment history.

\section{Microinjections}

Microinjections of drugs were administered 30 min before conditioning. In all experiments, the drug was injected before conditioning. During injections subjects were gently wrapped in a soft towel, the obturator was removed, and a 33-gauge microinjector (Plastics One) attached to PE50 tubing was inserted through the indwelling guide cannula. The distal end of the PE50 tubing was attached to a 10-1 syringe (Hamilton), which was attached to an infusion pump (Razel Scientific Instruments) that accurately dispensed the desired volume per side over a 2-min period. The microinjector remained in place for $1 \mathrm{~min}$ following injection. A volume of $1 \mu \mathrm{L}$ was bilaterally injected in all experiments.

\section{Drugs}

DHPG, (R.S.)-3,5-dihydroxyphenylglycine (Tocris), and AIDA, (R,S)-1-aminoindan-1,5-dicarboxylic acid (Tocris), were dissolved in saline solution for a final concentration of $50 \mu \mathrm{M}$ per side. Controls received equivolume of the vehicle (saline solution). In Experiment 3, DHPG and AIDA were dissolved in saline solution together for a final concentration of $50 \mu \mathrm{M}$ of each drug. In Experiment 4, DHPG and MPEP (Tocris) were dissolved in saline solution together for a final concentration of $50 \mu \mathrm{M}$ of DHPG and $200 \mu \mathrm{M}$ of MPEP.

\section{Histology}

At the completion of the experiment, rats were anesthetized with Nembutal $(50 \mathrm{mg} / \mathrm{kg}$ ) and decapitated, and their brains were removed and frozen in cold isopenthane. Coronal sections (40- $\mu \mathrm{m}$ thick) were taken through the amygdala with a cryostat at $-19^{\circ} \mathrm{C}$, and every third section was mounted. Sections were stained with cresyl violet and examined by light microscopy to visually verify the placement of the cannula in the BLA. Only rats with proper cannula placements were included in the analyses of each experiment. Cannula placements in the amygdala were verified by injecting a solution of $1 \%$ Evans Blue dye before decapitation. 
In all experiments the intent was to inject the drug into the BLA region. Rats were eliminated from the experiment if either of the cannula track marks was anterior to $-2.30 \mathrm{~mm}$ or posterior to $-3.6 \mathrm{~mm}$; lateral to $\pm 5.5 \mathrm{~mm}$ or medial to $\pm 4.5 \mathrm{~mm}$; or dorsal to $-7.0 \mathrm{~mm}$ or ventral to $-9.0 \mathrm{~mm}$.

\section{Acknowledgments}

We thank Karli Wright-Hardesty for technical assistance. This research was supported by National Institutes of Health grant NIH RO1 MH61316 to J.W.R.

\section{References}

Abraham WC. 2008. Metaplasticity: Tuning synapses and networks for plasticity. Nat Rev Neurosci 9: 387.

Abraham WC, Bear MF. 1996. Metaplasticity: The plasticity of synaptic plasticity. Trends Neurosci 19: 126-130.

Abraham WC, Tate WP. 1997. Metaplasticity: A new vista across the field of synaptic plasticity. Prog Neurobiol 52: 303-323.

Alarcon JM, Barco A, Kandel ER. 2006. Capture of the late phase of longterm potentiation within and across the apical and basilar dendritic compartments of CA1 pyramidal neurons: Synaptic tagging is compartment restricted. J Neurosci 26: 256-264.

Balschun D, Manahan-Vaughan D, Wagner T, Behnisch T, Reymann KG, Wetzel W. 1999. A specific role for group $1 \mathrm{mGluRs}$ in hippocampal LTP and hippocampus-dependent spatial learning. Learn Mem 6: 138-152.

Blanchard RJ, Blanchard DC. 1969. Crouching as an index of fear. J Comp Physiol Psychol 67: 370-375.

Bortolotto ZA, Fitzjohn SM, Collingridge GL. 1999. Roles of metabotropic glutamate receptors in LTP and LTD in the hippocampus. Curr Opin Neurobiol 9: 299-304.

Bradshaw KD, Emptage NJ, Bliss TVP. 2003. A role for dendritic protein synthesis in hippocampal late LTP. Eur J Neurosci 18: 3150-3152.

Cohen AS, Abraham WC. 1996. Facilitation of long-term potentiation by prior activation of metabotropic glutamate receptors. J Neurophysiol 76: 953-962.

Cohen AS, Raymond CR, Abraham WC. 1998. Priming of long-term potentiation induced by activation of metabotropic glutamate receptors coupled to phospholipase C. Hippocampus 8: 160-170.

Dudek SM, Fields RD. 2002. Somatic action potentials are sufficient for late-phase LTP-related cell signaling. Proc Natl Acad Sci 99: 39623967.
Fonseca R, Nägerl UV, Morris RG, Bonhoeffer T. 2004. Competing for memory: Hippocampal LTP under regimes of reduced protein synthesis. Neuron 44: 1011-1020.

Frey S, Frey JU. 2008. "Synaptic tagging" and "cross-tagging" and related associative reinforcement processes of functional plasticity as the cellular basis for memory formation. Prog Brain Res 169: 117-143.

Frey U, Morris RGM. 1997. Synaptic tagging and long-term potentiation. Nature 385: 533-536.

Frey U, Morris RG. 1998. Synaptic tagging: Implications for late maintenance of hippocampal long-term potentiation. Trends Neurosci 21: $181-188$.

Huber KM, Sawtell NB, Bear MF. 1998. Effects of the metabotropic glutamate receptor antagonist MCPG on phosphoinositide turnover and synaptic plasticity in visual cortex. J Neurosci 18: 1-9.

Kim J, Lee S, Park H, Song B, Hong I, Geum D, Shin K, Choi S. 2007. Blockade of amygdala metabotropic glutamate receptor subtype 1 impairs fear extinction. Biochem Biophys Res Commun 30: 188-193.

Lu YM, Jia Z, Janus C, Henderson JT, Gerlai R, Wojtowicz JM, Roder JC. 1997. Mice lacking metabotropic glutamate receptor 5 show impaired learning and reduced CA1 long-term potentiation (LTP) but normal CA3 LTP. I Neurosci 17: 5196-5205.

Paxinos G, Watson C. 1998. The rat brain in stereotaxic coordinates, 4 th ed. Academic Press, San Diego, CA.

Raymond CR, Thompson VL, Tate WP, Abraham WC. 2000. Metabotropic glutamate receptors trigger homosynaptic protein synthesis to prolong long-term potentiation. J Neurosci 20: 969-976.

Reymann KG, Frey JU. 2007. The late maintenance of hippocampal LTP: Requirements, phases, "synaptic tagging," "late-associativity" and implications. Neuropharm 52: 24-40.

Riedel G, Casabona G, Platt B, Macphail EM, Nicoletti F. 2000. Fear conditioning-induced time- and subregion-specific increase in expression of mGlu5 receptor protein in rat hippocampus. Neuropharm 39: 1943-1951.

Rodrigues SM, Bauer EP, Farb CR, Schafe GE, LeDoux JE. 2002. The group 1 metabotropic glutamate receptor mGluR5 is required for fear memory formation and long-term potentiation in the lateral amygdala. J Neurosci 22: 5219-5229.

Sajikumar S, Navakkode S, Frey JU. 2007. Identification of compartmentand process-specific molecules required for "synaptic tagging" during long-term potentiation and long-term depression in hippocampal CA1. J Neurosci 27: 5068-5080.

Steward O, Schuman EM. 2001. Protein synthesis at synaptic sites on dendrites. Annu Rev Neurosci 24: 299-325.

Sutton MA, Schuman EM. 2006. Dendritic protein synthesis, synaptic plasticity, and memory. Cell 127: 49-58.

Received April 5, 2009; accepted in revised form April 27, 2009. 


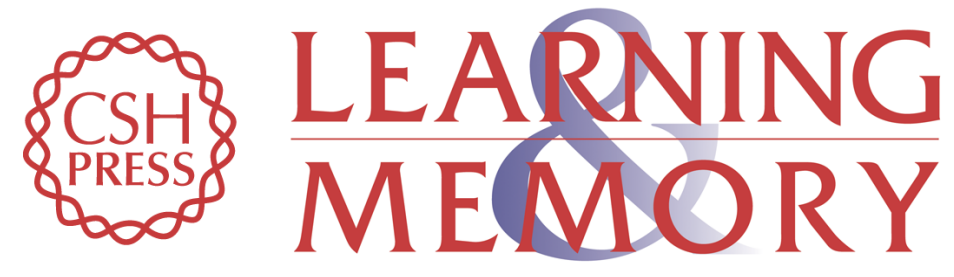

\section{DHPG activation of group 1 mGluRs in BLA enhances fear conditioning}

Jerry W. Rudy and Patricia Matus-Amat

Learn. Mem. 2009, 16:

Access the most recent version at doi:10.1101//m.1444909

Supplemental http://learnmem.cshlp.org/content/suppl/2009/06/29/16.7.421.DC1
Material

References This article cites 25 articles, 8 of which can be accessed free at:

http://learnmem.cshlp.org/content/16/7/421.full.html\#ref-list-1

License

Email Alerting Receive free email alerts when new articles cite this article - sign up in the box at the Service top right corner of the article or click here. 